\title{
Comparison between Conservative and Surgical Treatment in Proximal Humeral Fractures. A Prospective Randomized Study with 5-Years Follow-Up
}

\author{
M. Guzzini ${ }^{1}$, R. M. Lanzetti ${ }^{2}$, G. Princi ${ }^{1}$, M. Spoliti ${ }^{2}$, M. C. Moretti ${ }^{1}$, G. Koverech ${ }^{1}$, \\ A. P. Vadalà', D. Mazza ${ }^{1}$, F. Cannari², D. Topa², A. Ferretti ${ }^{1}$ \\ 1 Department of Trauma and Orthopaedics, Sant'Andrea Hospital, University of Rome La Sapienza, Rome, Italy \\ 2 Department of Trauma and Orthopaedics, San Camillo Hospital, Rome, Italy
}

\section{CORRESPONDING AUTHOR:}

Riccardo Maria Lanzetti

Department of Trauma and Orthopaedics

San Camillo Hospital

via Ginori 7

00100 Rome, Italy

E-mail: Riccardolanzetti@gmail.com

DOI:

10.32098/mltj.02.2021.05

LEVEL OF EVIDENCE: 2B

\begin{abstract}
SUMMARY
Background. There is no consensus on the optimal treatment of displaced proximal humeral fractures. The purpose of this prospective study was to present a 5 -year follow-up of conservative treatment of proximal humeral fractures as compared to surgical treatment (ORIF).

Materials and methods. Fifty consecutive patients with a proximal humeral fracture (Neer types 2, 3, 4; hertel types 3 through 8) were enrolled in this study and randomized in two groups: conservative treatment (group A) and open reduction and internal fixation (ORIF) with a plate (group B). Twenty patients of group A and 15 patients of group B were available for the final follow-up. All patients were clinically evaluated at 1,3 , and 12 months, and at final follow-up with physical and radiological examination, functional scores (Constant-Murley, quick DASH, ASES, SF-12), and overall satisfaction (from 1 to 4 ).

Results. The groups were homogeneous with regard to demographic data and radiological classifications of fractures. At the final follow-up (5.21 \pm 1.30 years), the Constant-Murley score was $82.0 \pm 7.6$ (group A) and $87.0 \pm 5.4$ (group B) (n.s.); quickDASH was $8.7 \pm 4.3$ (group A) and $5.4 \pm 0.37$ (group B) (n.s.); ASES was $83.7 \pm 7.0$ (group A) and $96.1 \pm 1.0$ (group B) $(\mathrm{p}=0.003)$; and SF-12 was $88.2 \pm 5.9$ (group A) and $90.1 \pm 5.7$ (group B) (n.s.). Eighty-five percent of patients in group A and 100\% in group B were satisfied or very satisfied with their results (n.s.). One patient in group $B$ reported wound dehiscence, which was treated with medical therapy.

Conclusions. Conservative treatment in proximal humeral fractures provides similar results in functional and symptom-related scores after 5 years, compared to ORIF. Indications for ORIF should be reserved only for patients with high functional demand.
\end{abstract}

\section{KEY WORDS}

Clinical outcomes; fracture; bumerus; nonoperative treatment; ORIF; plate; radiological outcomes.

\section{BACKGROUND}

Proximal humeral fractures represent $4-5 \%$ of all fractures, the second most common fracture of the upper extremity after forearm fractures (1), and the third most common fracture in the elderly after Colles' and hip fractures (2). Female sex and osteoporosis are the most important risk factors (3). In $87 \%$ of cases, fractures occur after low-energy trauma $(4,5)$.
There are different classification systems for proximal humeral fracture. The Neer classification (1970) (6) is probably the most frequently used, it divides fractures depending on an anatomic segmental classification. It is globally accepted that two-part fractures with minimal displacement can be treated conservatively. During the $8^{\text {th }}$ ICSS (International Conference on Shoulder Surgery), 
in 2001, Hertel presented a morphologic classification of proximal humeral fractures using a binary description system in which five basic planes were identified. Through a combination of blocks he managed to recognize 12 fracture patterns: between the greater tuberosity and the head, the greater tuberosity and the shaft, the lesser tuberosity and the head, the lesser tuberosity and the shaft, the lesser tuberosity and the greater tuberosity, six dividing the humerus into two fragments, five dividing the humerus into three fragments, and a single fracture dividing the humerus into four fragments (7).

Minimally displaced proximal humeral fractures could be treated nonoperatively; the treatment of displaced fractures is controversial and includes conservative treatment (immobilization with cast, Sling and Swathe brace or similar), percutaneous pinning, intramedullary nailing, open reduction and internal fixation (ORIF) with locking plate, and arthroplasty (8-14).

The aim of this study is to evaluate at 5 -year follow-up, the clinical and radiological outcomes of conservative treatment of proximal humeral fracture types 2, 3, and 4 (not displaced in varus) according to the Neer classification and types 3 through 8 of Hertel's system, compared to surgical treatment (ORIF with plate). The hypothesis is that there are no differences in clinical and radiological outcomes at final follow-up for these types of fractures.

\section{MATERIALS AND METHODS}

Eighty consecutive patients aged between 50 and 65 years who suffered proximal humeral fracture between 2013 and 2014 were enrolled in this prospective study. Each fracture was classified according to the Neer and Hertel classifications and was labeled in the study as one or more of the following fracture types: Neer type 2, 3, or 4 (not displaced in varus) and hertel types 3 through 8 .

Exclusion criteria were the following: history of proximal humeral fracture; previous local and general infective disease; and previous surgery on the affected shoulder. After inclusion and exclusion criteria were applied, patients available for the study were randomly divided in two groups: group A, conservative group; and group B, surgical group.

The present study was approved by the ethics committees of both hospitals involved, in accordance with the ethical standards of the journal (15).

\section{Conservative treatment protocol}

Patients in group A were treated with Desault bandage immobilization for the first week. Shoulders were then immobilized with a Sling and Swathe shoulder brace from week 2 through week 4 . At one month, mobilization was allowed after controlling bone healing with X-rays.

\section{Surgical treatment protocol}

The surgery was performed by the same surgeon for all patients of group B: the patient was placed in supine position; a deltopectoral approach was used; the fracture was identified and periosteum was debrided back from the fracture edges by $1-2 \mathrm{~mm}$, just far enough for clear visualization of the fracture; reduction was achieved by using pointed reduction clamps, then a plate of sufficient length was fixed using screws. In the post-operative protocol, patients' shoulders were immobilized with an abduction shoulder brace immediately after the surgery. At the 2 -week clinical check, the shoulder brace was removed, and patients were encouraged to perform Codman's pendulum exercises. An X-ray was performed after one month and full movement authorized, in the absence of contraindications.

\section{Radiographic evaluation}

Radiographic evaluation was performed obtaining both standard orthogonal planes of the anteroposterior shoulder and a lateral view of the scapula. Patients in group B were evaluated by X-ray postoperatively, and patients in both groups were evaluated at one week, at one, three, and twelve months; and at final follow-up (figure 1).

\section{Clinical outcomes}

All patients were clinically evaluated at one, three, and twelve months and at final follow-up with a physical examination. The Constant-Murley Score (CMS) (16), the Disabilities of the Arm, Shoulder and Hand Questionnaire (quickDASH) (17), the American Shoulder and Elbow Surgeons Shoulder Score (ASES) (18), and the Short-Form 12 (SF-12) health survey (19) were recorded at the final follow-up, along with a patient satisfaction rating: (1: disappointed; 2 : moderately satisfied; 3: satisfied; 4: very satisfied).

\section{Statistical analysis}

Before treatment, patients were randomized into two groups (figure 2) through computer-generated blocked-randomization numbers (http://www.randomizer.org). All patients were informed of the advantages and disadvantages of both treatments. SPSS 22.0 (IBM, Armonk, NY, USA) was used for data analysis. Descriptive statistics were used to 


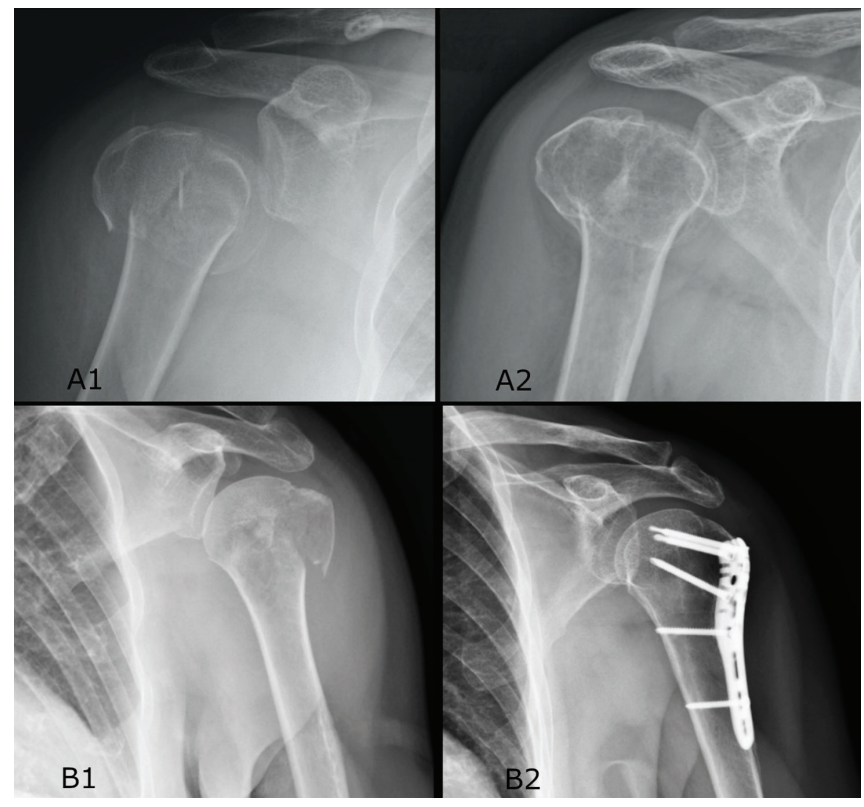

Figure 1. X-rays. (A1) Conservative group fracture, (A2) conservative group fracture at final follow-up; (B1) surgical group preoperative, (B2) surgical group at final follow-up.

summarize characteristics of the study patients (i.e., score results), including means and ranges. The Wilcoxon Test was used to detect significant differences in the evaluated scores at final follow-up. Results were compared with the Mann-Whitney U Test. Statistical significance was set at a p-value $<0.05$.

\section{RESULTS}

From the initial sample, 20 patients of group A and 15 patients of group B were available for the final follow-up (figure 2). The two groups were homogeneous with regard to age, gender, and dominance: group A was composed of eleven women and nine men (mean age, $60.45 \pm 1.9$ ); group

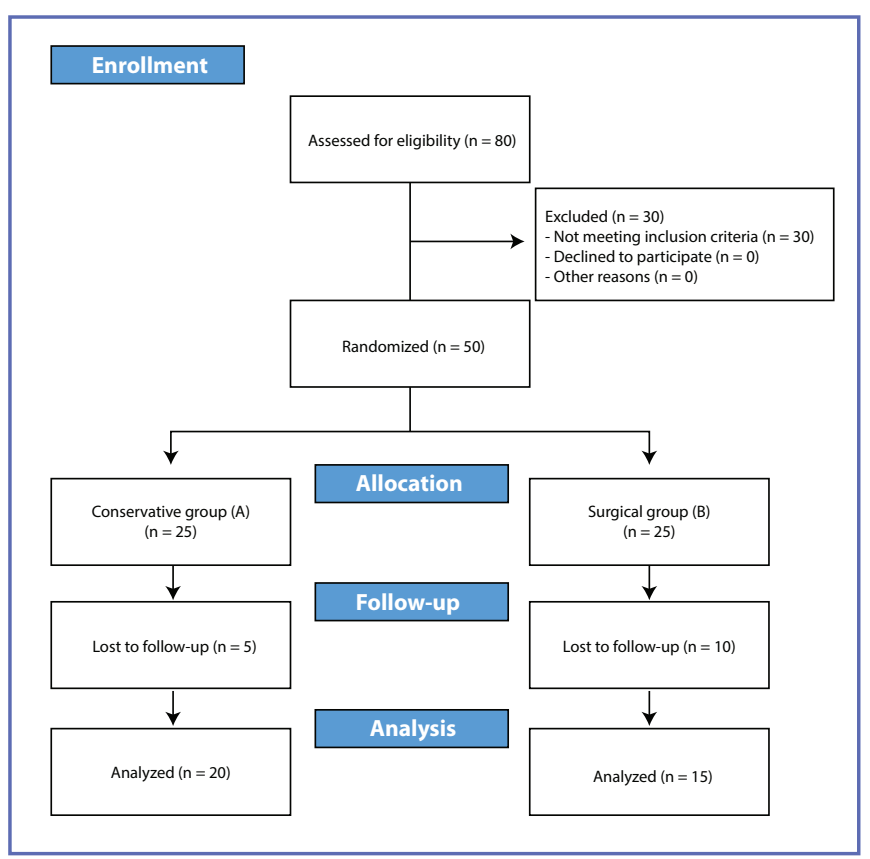

Figure 2. Flowchart.

B was composed of nine women and six men (mean age, $55.40 \pm 3.48$ ). Demographic data intergroup comparison were reported in table I. The mean follow-up was $5.11 \pm$ 1.30 years: $5.25 \pm 1.34$ years for group $\mathrm{A}$, and $4.93 \pm 1.24$ years for group B $(\mathrm{p}=0.143)$.

All fractures were classified according to the Neer's and Hertel's classifications, as summarized in table II. No statistically significant differences, based on fracture classifications, were found between the two groups.

Clinical results at final follow-up showed: a median CMS of $82.0 \pm 7.6$ in group $A$, and $87.0 \pm 5.4$ in group $B(p=0.166)$; a median quickDASH of $8.7 \pm 4.3$ in group $\mathrm{A}$, and $5.4 \pm 0.4$ in group $B(p=0.101)$; a median ASES of $83.7 \pm 7.0$ in group $A$, and $96.1 \pm 1.0$ in group B $(p=0.003)$; and a median SF-12 of $88.2 \pm 5.9$ in group $A$, and $90.1 \pm 5.7$ in group $B(p=0.327)$.

Table I. Demographic table. Intergroup comparison.

\begin{tabular}{llll}
\hline Variable & Group A (Conservative) $\mathbf{n}=\mathbf{2 0}$ & Group B $($ ORIF) $\mathbf{n}=\mathbf{2 6}$ & $\mathbf{p}^{\mathbf{b}}$ \\
\hline Follow-up \pm SD & $5.25 \pm 1.34$ & $4.93 \pm 1.24$ & 0.143 \\
\hline Average age \pm SD & $60.5 \pm 1.9$ & $55.4 \pm 3.5$ & 0.015 \\
\hline Gender (Male/Female) & $9 / 11$ & $6 / 9$ & 0.409 \\
\hline Dominant side (Right/Left) & $18 / 2$ & $14 / 1$ & 0.440 \\
\hline Affected side (Right/Left) & $9 / 11$ & $8 / 7$ & 0.345 \\
\hline Complications & 0 & 1 & 0.374 \\
\hline
\end{tabular}

SD: Standard Deviation; 'boldface indicates statistical significance $(\mathrm{p}<0.05)$. 
Table II. Fracture classification. Intergroup comparison.

\begin{tabular}{|c|c|c|c|c|}
\hline Variable & & Group A (Conservative) $n=20$ & Group B (ORIF) n = 15 & $\mathbf{p}^{\mathrm{b}}$ \\
\hline Neer Classification & $\begin{array}{l}2 \\
3 \\
4\end{array}$ & $\begin{array}{l}11(55 \%) \\
8(40 \%) \\
1(5 \%)\end{array}$ & $\begin{array}{l}5(33 \%) \\
6(40 \%) \\
4(27 \%)\end{array}$ & 0.067 \\
\hline Hertel Classification & $\begin{array}{l}1 \\
2 \\
3 \\
4 \\
5 \\
6 \\
7 \\
8\end{array}$ & $\begin{array}{l}1(5 \%) \\
0(0 \%) \\
4(20 \%) \\
1(5 \%) \\
4(20 \%) \\
0(0 \%) \\
4(20 \%) \\
6(30 \%)\end{array}$ & $\begin{array}{l}0(0 \%) \\
0(0 \%) \\
1(7 \%) \\
3(20 \%) \\
3(20 \%) \\
2(13 \%) \\
3(20 \%) \\
3(20 \%)\end{array}$ & 0.500 \\
\hline
\end{tabular}

${ }^{\mathrm{b} B o l d f a c e}$ indicates statistical significance $(\mathrm{p}<0.05)$.

Seventeen patients of group A ( $85 \%)$ and 15 patient of group B $(100 \%)$ were satisfied or very satisfied $(p=0.058)$.

However, the results showed statistical significance only for ASES $(p=0.003)$. No statistical significance for the CMS (n.s.), quickDASH (n.s.), SF-12 (n.s.), or the Satisfaction rate (n.s.). Clinical data and intergroup comparison are reported in table III and IV.

One patients of group B reported wound dehiscence, which was successfully treated with medical therapy, and completely healed within four weeks of surgery. No other complications were reported. All patients in both groups showed bone healing without displacement and/or malunion.

\section{DISCUSSION}

The most significant finding of this study was that, with regard to the treatment of proximal humeral fractures, there was no difference in outcomes between conservative and surgical treatment with conventional stainless steel plates. In accordance with the literature, outcomes showed a substantial equivalence of clinical and radiographic results between the two groups at 5.11 years of follow-up. In fact a 2015 review by Xie et al. (20), which included nine articles and 518 patients, pointed out that operative treatment did not significantly improve the functional outcome and health-related quality of life.

Table III. Clinical results. Intergroup comparison.

\begin{tabular}{lccl}
\hline Variable $(\mathbf{\pm}$ SD) & Group A (Conservative) $\mathbf{n}=\mathbf{2 0}$ & Group B (ORIF) $\mathbf{n}=\mathbf{1 5}$ & $\mathbf{p}^{\mathrm{b}}$ \\
\hline Constant-Murley Score & $82.0 \pm 7.6$ & $87.0 \pm 5.4$ & 0.166 \\
\hline quickDASH & $8.7 \pm 4.3$ & $5.4 \pm 0.4$ & 0.101 \\
\hline ASES Shoulder Score & $83.7 \pm 7.0$ & $96.1 \pm 1.0$ & 0.003 \\
\hline SF-12 & $88.2 \pm 5.9$ & $90.1 \pm 5.7$ & 0,327 \\
\hline Satisfaction (1-4) & $3.0 \pm 0.5$ & $3.5 \pm 0.3$ & 0.058 \\
\hline
\end{tabular}

SD: Standard Deviation; ASES: American Shoulder and Elbow Surgeons; SF-12, Short Form-12: bboldface indicates statistical significance ( $<<0.05)$.

Table IV. Clinical results, Neer Classification subgroups. Intergroup comparison.

\begin{tabular}{|c|c|c|c|c|c|c|c|}
\hline Variable $( \pm$ SD $)$ & Group A (Con & rvative) $n=20$ & & Group B (OR & IF) $n=15$ & & $\mathbf{p}^{\mathrm{b}}$ \\
\hline & Neer $2 \mathrm{n}=11$ & Neer $3 n=8$ & Neer $4 n=1$ & Neer $2 n=5$ & Neer $3 n=6$ & Neer $4 n=4$ & \\
\hline Constant-Murley Sc. & $86.9 \pm 7.8$ & $79.4 \pm 12.0$ & $49.0 \pm \mathrm{NA}$ & $95.0 \pm 5.4$ & $83.2 \pm 8.3$ & $82.8 \pm 8.1$ & 0.166 \\
\hline quickDASH & $6.6 \pm 4.8$ & $9.7 \pm 6.9$ & $25.0 \pm \mathrm{NA}$ & $5.6 \pm 0.4$ & $5.0 \pm 0.7$ & $5.8 \pm 0.4$ & 0.101 \\
\hline ASES Shoulder Sc. & $87.9 \pm 9.1$ & $81.0 \pm 9.2$ & $59.9 \pm \mathrm{NA}$ & $96.4 \pm 2.0$ & $94.8 \pm 0.3$ & $97.8 \pm 1.8$ & 0.003 \\
\hline SF-12 & $92.5 \pm 6.3$ & $85.1 \pm 9.1$ & $66.0 \pm \mathrm{NA}$ & $93.0 \pm 2.7$ & $90.1 \pm 3.5$ & $86.4 \pm 1.6$ & 0,327 \\
\hline Satisfaction (1-4) & $3.2 \pm 0.5$ & $2.9 \pm 0.8$ & $2.0 \pm \mathrm{NA}$ & $3.4 \pm 0.4$ & $3.5 \pm 0.4$ & $3.5 \pm 0.5$ & 0.058 \\
\hline
\end{tabular}

SD: Standard Deviation; ASES: American Shoulder and Elbow Surgeons; SF-12: Short Form-12; bboldface indicates statistical significance (p < 0.05); NA: not applicable. 
In this study, there was only a slight difference with regard to the clinical evaluation forms between the two groups, yielding better results for the ORIF group. Again, in accordance with the literature, no statistically significant differences were found in the Costant-Murley and QuickDASH scores, or in the SF12 Questionnaire. On the other hand, the ASES score showed statistically significant outcomes; these results were correlated with a recovery of normal daily activities with both treatments. Patients with fractures in the dominant limb showed better functional outcomes, but this data is not relevant in this study since the two groups were homogeneous. The ORIF group, due to an early rehabilitation protocol, showed better outcomes in high demand activities.

No differences between the two treatments were otherwise found in the radiographic evaluation. Bone healing was achieved in all patients.

Only one patient reported a complication (surgical wound dehiscence), which could have been attributed this to the small sample included in the study.

The limits of conservative treatment are: prolonged immobilization, and therefore the risk of articular stiffness; avascular necrosis; and malunion and/or nonunion. In the literature, these results are supported by several studies, such as the 2014 review by Mao et al. (21) and the 2018 review by Reinier B. Beks et al. (22).

The former review included six studies and 287 patients, 144 of which were treated with conservative management: nonunion was identified in $5.47 \%(7 / 128)$; osteoarthritis in $14.6 \%$ (7/48); and avascular necrosis in $15.5 \%$ (20/128) of patients treated nonoperatively.

The latter review included twenty-two studies and 1743 patients, 833 of which were treated conservatively. Specifically, 13 studies demonstrated a nonunion rate of $5.6 \%$ (25/45), and a rate of $8.0 \%$ (35/439) of avascular necrosis. In five studies, $4.8 \%$ of patients (20/457) underwent revision surgery due to impingement, displacement, or malunion.

Even though surgical patients presented better functional outcomes, they ran into a higher incidence of infections, nerve injuries, and reintervention.

In a 2015 review by Xie et al. (20), operative and nonoperative treatments were compared. They reported that the surgical group had an infection rate five times higher than that of the non-surgical group; a nerve injury rate 1.57 times higher; and twice as high of reintervention and mortality rates, whereas, the rate of nonunion and displacement was reduced to almost half.

Moreover, as stated by Panagiotopoulou et al. (23) in their 2019 review, and also by McMillan \& Johnstone (24) in their 2018 review, implant-related complications such as screw perforations, screw cut-out, mobilization and plate bending, and break-age should not be overlooked.

In agreement with the significant rate of complications related to ORIF reported in the literature (25), this study suggests that the conservative approach leads to good clinical results and bone healing, and that it furthermore avoids the risks associated with surgery.

On the other hand, patients with high activity demand could prefer the surgical option since operative treatment could lead to a faster recovery.

In accordance with the 2011 study by Olerud et al. (26), this study suggested that the choice of treatment in patients with proximal humeral fractures should be based on functional demands and comorbidities of the patients.

The limitations of the study included the number of patients admitted to the study and the absence of comparison to other surgical treatments. Moreover, the follow-up period allowed us to draw conclusions only on the medium-term outcomes.

\section{CONCLUSIONS}

Conservative treatment is the gold standard for nondisplaced and minimally displaced proximal humeral fractures, whereas, in cases of majorly displaced humeral fractures, the treatment of choice in literature has yet to be defined.

This prospective study showed that the comorbidity and functional demand of patients must be taken into consideration in the management of majorly displaced proximal humeral fractures.

Since the clinical and radiological outcomes in conservatively treated patients are comparable to the outcome of patients treated with ORIF, conservative treatment is therefore a viable option for patients with low functional demand, and furthermore, the associated risks of surgery are avoided. Operative treatment is best suited for younger and healthy patients with higher functional demand, where early mobilization of the affected limb can lead to faster recovery for activities demanding higher performance.

\section{ETHICS}

The study protocol was approved by the hospitals' Ethical Review Board and it was conducted in accordance with the principles of the Declaration of Helsinki and its amendments. Patients were informed about the characteristics of the study and they gave their consent to participate.

\section{CONSENTS}

Consent was obtained from patients for publication of the study. No personal data was included in the manuscript. 


\section{CONTRIBUTIONS}

All authors contributed equally to the manuscript and read and approved the final version of the manuscript.

\section{REFERENCES}

1. Court-Brown CM, Caesar B. Epidemiology of adult fractures: A review. Injury 2006;37:691-7.

2. Rouxa A, Decroocqa L, El Battia S, et al. Epidemiology of proximal humerus fractures managed in a trauma center. Orthop Traumatol Surg Res 2012;98:715-9.

3. Bahrs C, Stojicevic T, Blumenstock G, et al. Trends in epidemiology and patho-anatomical pattern of proximal humeral fractures. Int Orthop 2014;38(8):1697-704.

4. Bell JE, Leung BC, Spratt KF, et al. Trends and variation in incidence, surgical treatment, and repeat surgery of proximal humeral fractures in the elderly. J Bone Joint Surg Am 2011;93(2):121-31.

5. Court-Brown CM, Garg A, McQueen MM. The epidemiology of proximal humeral fracture. Acta Orthop Scan 2001;72(4):365-71.

6. Brorson S, Bagger J, Sylvest A, Hrobjartsson. A Diagnosing displaced four-part fractures of the proximal humerus: a review of observer studies. Int Orthop 2009;33(2):323-7.

7. Hertel R, Hempfing A, Stiehler M, Leunig M. Predictors of humeral head ischemia after intracapsular fracture of the proximal humerus. J Shoulder Elbow Surg 2004;13(4):427-33

8. Misra A, Kapur R, Maffulli N. Complex proximal humeral fractures in adults - a systematic review of management. Injury 2001;32:363-72.

9. Keser S, Bölükbasi S, Bayar A, Kanatlı U, Meray J, Özdemir H. Proximal humeral fractures with minimal displacement treated conservatively. Int Orthop 2004;28:231-4.

10. Shrader MW, Sanchez-Sotelo J, Sperling JW, Rowland CM, Cofield RH. Understanding proximal humerus fractures: image analysis, classification, and treatment. J Shoulder Elbow Surg 2005;14(5):497-505.

11. De la Hoz Marín J, Hernández Cortés P, Tercedeor Sánchez J. Surgical treatment of three-part proximal humeral fractures. Acta Orthop Belg 2001;67(3):226-32

12. Fenichel I, Oran A, Burstein G, Perry Pritsch M. Percutaneous pinning using threaded pins as a treatment option for unstable two- and three-part fractures of the proximal humerus: a retrospective study. Int Orthop 2006;30(3):153-7.

13. Savin DD, Zamfirova I, Iannotti J, Goldberg BA, Youderian AR. Survey study suggests that reverse total shoulder arthroplasty is becoming the treatment of choice for fourpart fractures of the humeral head in the elderly. Int Orthop 2016;40:1919-25.

14. Sun Q, Ge W, Li G, et al. Locking plates versus intramedullary nails in the management of displaced proximal humeral fractures: a systematic review and meta-analysi. Int Orthop 2018;42(3):641-50.

\section{CONFLICT OF INTERESTS}

The authors declare that they have no conflict of interests.

15. Padulo J, Oliva F, Frizziero A, Maffulli N. Muscles, Ligaments and Tendons Journal - Basic principles and recommendations in clinical and field Science Research: 2018 Update. Muscles Ligaments Tendons J 2018;8(3):305-7.

16. Roy JS, MacDermid JC, Woodhouse LJ. A systematic review of the psychometric properties of the Constant-Murley score. J Shoulder Elbow Surg 2010;19:157-64.

17. Hudak P, Amadio PC, Bombardier C, and the Upper Extremity Collaborative Group. Development of an Upper Extremity Outcome Measure: The DASH (Disabilities of the Arm, Shoulder, and Hand). Am J Ind Med 1996;29:602-08.

18. Kirkley A, M, Griffin S, Dainty K. Scoring Systems for the Functional Assessment of the Shoulder. ISAKOS Scientific Committee Report 2003;19(10):1109-20.

19. Ware J, Kosinski M, Keller SD. A 12-item short-form health survey: construction of scales and preliminary tests of reliability and validity. Med Care 1996;34:220-33.

20. Xie L, Ding F, Zhao Z, Chen Y, Xing D. Operative versus non-operative treatment in complex proximal humeral fractures: a meta-analysis of randomized controlled trials. Springerplus 2015;4(1):728.

21. Mao Z, Zhang L, Zhang L, et al. Operative versus nonoperative treatment in complex proximal humeral fractures. Orthopedics 2014;37(5):e410-9.

22. Beks RB, Ochen Y, Frima H, et al. Operative versus nonoperative treatment of proximal humeral fractures: a systematic review, meta-analysis, and comparison of observational studies and randomized controlled trials. J Shoulder Elbow Surg 2018;27(8):1526-34.

23. Panagiotopoulou VC, Vargaa P, Richardsa RG, Gueorguieva B, Giannoudisc PV. Late screw-related complications in locking plating of proximal humerus fractures: A systematic review. Injury 2019;50:2176-95.

24. McMillan TE, Johnstone AJ. Primary screw perforation or subsequent screw cut-out following proximal humerus fracture fixation using locking plates: a review of causative factors and proposed solutions. Int Orthop 2018;42(8):1935-42

25. Song JQ, Deng XF, Wang YM, Wang XB, Li X, Yu B. Operative vs. nonoperative treatment for comminuted proximal humeral fractures in elderly patients: a current meta-analysis. Acta Orthop Traumatol Turc 2015;49(4):345-53.

26. Olerud P, Ahrengart L, Ponzer S, Saving J, Tidermark J. Internal fixation versus nonoperative treatment of displaced 3-part proximal humeral fractures in elderly patients: a randomized controlled trial. J Shoulder Elbow Surg 2011;20(5):747-55. 